\title{
MODERN DIAGNOSIS OF PLACENTAL DYSFUNCTION AND ITS COMPLICATIONS
}

\author{
Lazurenko V.V., Borzenko I.B., Lyashchenko O.A., \\ Ovcharenko O.B., Tertyshnyk D.Yu. \\ Kharkiv National Medical University \\ https://doi.org/10.35339/ic.8.3.182-187
}

\begin{abstract}
The aim of the study was to improve the modern diagnosis of placental dysfunction and its complications. Materials and methods. The study involved a prospective survey of 70 pregnant women divided into the main group (pregnant women with placental dysfunction) $(\mathrm{n}=50)$ and the control group $(n=20)$. The main group was divided into subgroups of pregnant women with placental dysfunction and fetal growth retardation $(n=30)$ and pregnant women with placental dysfunction without fetal growth retardation $(n=20)$. The control group comprised 20 pregnant women with physiological gestation. Apart from history taking, the study comprised obstetric and general clinical examination, evaluation of endotheliumdependent vasodilation, serum concentrations of soluble forms of vascular and plateletendothelial molecules of cell adhesion 1, indicators of athrombogenicity of the vascular growth wall, uterine-placental-fetal blood circulation, pathomorphological and histometric examination of the placenta. Results. Based on the obtained clinical-morphological and endotheliotropic criteria, a personalized clinical algorithm for managing pregnant women with placental dysfunction was developed and implemented. Conclusions. Assessment of pregnancy results in a prospective clinical study showed that the proposed algorithm for personalization of the risk of perinatal abnormalities not only helped to avoid antenatal mortality, but also to prevent intranatal and early neonatal losses in patients with placental dysfunction and fetal growth retardation.
\end{abstract}

Keywords: gestational endotheliopathy, fetal growth retardation, placental dysfunction, personalized diagnostic algorithm.

\section{Introduction}

Placental dysfunction (PD) remains one of the pressing problems of obstetrics.

According to the literature, the incidence of placental dysfunction (PD) in habitual miscarriage ranges from 50 to $77 \%$, in preeclampsia this figure reaches more than $65 \%$, in pregnancy complicated by extragenital abnormality it occurs in 24 $45 \%$, in pregnant women with viral or bacterial infections in more than $60 \%$ of cases [1-5]. Placental dysfunction is the cause of fetal distress, fetal growth retardation (FGR), abnormal conditions and diseases of the newborn. According to the WHO, the number of newborns with growth retardation ranges from $6.5 \%$ in the developed

Corresponding Author:

Viktoriya Lazurenko MD, PhD, Professor, Head

of Department of Obstetrics and Gynecology No.2, Kharkiv National Medical University, Ukraine.

E-mail: vv.lazurenko@knmu.edu.ua
European countries to $31.1 \%$ in Central Asia. In the US, FGR occurs in every $10-15 \%$ of births; meanwhile, severe intranatal hypoxia is observed in $30 \%$. Perinatal mortality in pregnant women with placental dysfunction is $8-15 \%$ among fullterm infants and 40-50\% among premature infants. Placental dysfunction results in the formation of fetal growth retardation syndrome in $60-70 \%$ of cases $[1,6-10]$.

Impaired placental function is due to cardiovascular diseases in pregnant (heart disease, circulatory failure, hypertension and hypotension), chronic infection, disorders of the lungs, liver, kidneys, blood, diseases of the endocrine system (diabetes, hypo- and hyperthyroidism), obstetric and gynecological diseases, harmful habits, social and household factors [1, 11].

Primary PD begins to develop in early pregnancy (up to 14-18 weeks) in the early stages of placental development and embryogenesis, under the influence of genetic, endocrine, infectious and 
environmental factors. Enzymatic insufficiency of decidual tissue (in luteal phase insufficiency), defects of structure and localization of the placenta, as well as impairment of vascularization and disturbance of chorion morphology play an important role in the development of primary PD. Patients with primary PD are more often found to have fetal malformations, chromosomal abnormalities and intrauterine infection [12-14].

Secondary placental dysfunction can develop secondarily to the already formed placenta after 18 weeks of pregnancy under the influence of exogenous factors. The morphological substrate of secondary PD is a violation of uteroplacental and fetoplacental blood flow, as well as related dystrophic changes of the chorionic villi, combined with cellular and tissue compensatory reactions of the placenta [15].

According to its clinical course, PD is divided into acute and chronic. Acute PD develops with a sudden violation of decidual perfusion and adequate hemodynamic support of pregnancy. Morphological examination reveals hemorrhages in the placenta. Acute PD can occur at any stage of pregnancy in the form of premature detachment of the normally located placenta, during childbirth and is the cause of acute distress and fetal death.

Chronic PD is observed in every third pregnant woman with a high risk of perinatal impairment, its course is longer due to impaired adaptive mechanisms in combination with circulatory disorders, involutive changes that may be associated with extragenital disorders in women during pregnancy $[16,17]$.

Methods of assessment of the fetoplacental complex are used in due time to diagnose placental disfunction and FGR. Prenatal diagnosis of these conditions includes Doppler ultrasound (US), cardiotocography (CTG), placental hormones (estriol, cortisol, placental lactogen, progesteron) $[1,18-21]$. The content of vascular-endothelial growth factor, placental growth factor, interleukin-1, interleukin-1, endothelin-1, pulsation index of spiral arteries, umbilical arteries and uterine arteries are also considered to be early markers of PD prediction $[4,13,19]$.

Despite the introduction of new methods of diagnosis and prevention of placental dysfunction into obstetric practice, there is no clear tendency to decrease its incidence. Therefore, it is important to use the latest research methods as prognostic criteria for placental dysfunction and its manifest form like FGR, which will improve the prognosis of gestation, develop an optimal strategy for the term and mode of delivery and, as a consequence, prevent the development of obstetric and perinatal pathology $[1,4]$.

\section{Purposes, Subjects and Methods}

2.1. Purpose of the study was to optimize the modern diagnosis of placental dysfunction and its complications.

\subsection{Subjects \& Methods}

The study was conducted by Department of Obstetrics and Gynecology No.2 of Kharkiv National Medical University at Kharkiv Regional Perinatal Centre (2016-2020). The first stage of the study was a retrospective assessment of 100 individual pregnancy records of women with PD to determine the risk factors for the development of placental dysfunction. The second stage included prospective survey of 70 pregnant women, who were divided into the main group (50 pregnant women with PD) and the control group (20 pregnant women with physiological pregnancy). The main group was divided into groups of 20 pregnant women with $\mathrm{PD}$ and 30 pregnant women with $\mathrm{PD}$ and FGR.

The examination of women included history taking, general clinical examination, ultrasonography of the fetus and placenta, in particular, Doppler imaging of the uteroplacental complex, as well as homeostasis indicators such as vascular endothelial growth factor (VEGF), platelet-endothelial molecules of cell adhesion 1 (sVCAM and sPECAM), indicators of atrombogenicity of the vascular wall (thrombomodulin, thrombospondin, tissue plasminogen activator and plasminogen activator inhibitor-1), antioxidant (MDA, DC, catalase and SOD) and lipid peroxidation system. Morphological examination of the placenta was carried out [1]. Statistical processing of the study results was performed using the statistical analysis software Microsoft Excel and Statistica-6.0 (Statsoft), applying parametric and non-parametric methods for evaluating the results.

\section{Results \& Discussion}

A study of social and household conditions showed that PD more often developed in patients who had low socio-economic status $(\mathrm{p}=0.0002)$, smoked for 5-8 years $(\mathrm{p}=0.0001)$, spent more than 5 hours a day working on the computer $(\mathrm{p}=0.04)$, led a sedentary lifestyle $(\mathrm{p}=0.04)$. $17.5 \%$ of women with placental dysfunction continued to smoke during their current pregnancy. Besides, $53.8 \%$ of women in the main group indicated that they had drunk soft alcoholic drinks before pregnancy [1].

The structure of extragenital morbidity in pregnant women with PD was associated with 
the varicose veins $(\mathrm{p}=0.02)$, cardiovascular $(\mathrm{p}=$ 0.05 ), gastrointestinal diseases, namely, dyskinesia of the biliary tract $(\mathrm{p}=0.03)$, infectious diseases $(p=0.04)$. An important place in the development of PD was also occupied by hereditary family history, namely cases of arterial hypertension $(p=0.02)$, endocrine disorders $(p=0.02)$, thrombotic complications $(p=0.02)$ [1].

Assessment of the obstetric history of women with PD showed that previous pregnancies in women with placental pathology were complicated by spontaneous abortions $(\mathrm{p}=0.03)$, induced abortions $(p=0.002)$, disorders of the ovarianmenstrual cycle $(p=0.04)$ and disorders of vaginal microbiocenosis $(p=0.03)$. Obstetric disorders included preterm birth $(p=0.04)$ and threatening miscarriage $(p=0.008)$. In general, the threat of miscarriages may be evaluated as an integral indicator of the clinical manifestation of inferiority of cytotrophoblast invasion, which may subsequently lead to PD [1].

Evaluation of endotheliotropic vasotonic properties in the examined pregnant involved employment of a test with reactive hyperemia of the brachial and posterior tibial arteries, applying high resolution ultrasound - an indicator of endothelium-dependent vasodilation. Pregnant women with manifested course of placental dysfunction, namely, fetal growth retardation, were found to have critical disorders of vasoregulatory function of the endothelium in both examined vessels, with a clear reliable vasoconstrictor component at the 28-40 weeks of pregnancy, even in comparison with women who had preclinical PD $(\mathrm{p}<0.05)$.

Pregnant women with PD and FGR in gestational endotheliopathy had an increase in serum indicators of VEGF $(5.89 \pm 0.31 \mathrm{pg} / \mathrm{ml})$. Serum thrombospondin concentration in patients with FGR increased by 2.2 times $(3.37 \pm 0.40 \mathrm{pg} / \mathrm{ml})$, compared with those in pregnant women of the control group and compared with the results of women with preclinical significant form of PD $(\mathrm{p}<0.05)$.

In addition to assessing the content of major vasoconstrictors and vasodilators, the functional state of the vascular endothelium allowed assessment of the level of soluble molecules of intercellular adhesion. I.B. Borzenko observed an increase in the level of soluble forms of vascular cell adhesion molecules 1 (sVCAM-1) to $1721.72 \pm 161.63 \mathrm{ng} / \mathrm{ml}$ in serum during placental dysfunction, which was complicated by fetal growth retardation compared to similar indicators during physiological pregnancy $-796.49 \pm 75.90 \mathrm{ng} / \mathrm{mg}$ and donological placental dysfunction $-1065.78 \pm$
$128.24 \mathrm{ng} / \mathrm{ml}(\mathrm{p}<0.05)$. Assessment of the results of sPECAM -1 presence in the serum of pregnant women with placental dysfunction showed a decrease in the concentration of soluble form of the above-mentioned MKA compared with the samples obtained from almost healthy pregnant respondents $(96.4 \pm 7.9 \mathrm{ng} / \mathrm{ml})$. However, we did not observe a statistically significant difference $(p>0.05)$ between serum levels of sPECAM- 1 in women who had complicated placental dysfunction $(68.4 \pm 7.3 \mathrm{ng} / \mathrm{ml})$ and those diagnosed with non-manifest placental dysfunction $(78.4 \pm 5.1 \mathrm{ng} / \mathrm{ml})$. An increase in the concentration of sVCAM-1 with simultaneous significant reducing SPECAM-1 during placental dysfunction may evidence endothelial dysfunction, which may be useful for stratifying the risk of manifestation of perinatal disorder of placental origin, namely fetal growth retardation [1].

The study of serum thrombomodulin (the thrombin receptor expressed on endothelial cell membranes) showed that there was a significant increase in the indicated marker of vascular thrombogenicity in women with placental dysfunction, compared to the results of practically healthy pregnant women $(4.28 \pm 0.47 \mathrm{ng} / \mathrm{ml})$. However, a statistically significant difference $(p<0.05)$ was observed in patients diagnosed with fetal growth retardation $(7.92 \pm 0.84 \mathrm{ng} / \mathrm{ml})$, not only in women with physiological pregnancy, but also in practically healthy pregnant women, who did not have any clinical manifestation of placental dysfunction $(5.25 \pm 0.53 \mathrm{ng} / \mathrm{ml})$ [1].

The study of tissue plasminogen activator in patients had a significant difference $(p<0.05)$ between the results of tissue plasminogen activator in pregnant women with physiological gestational course and patients with fetal growth retardation. At the same time, there was no statistically significant difference between serum tissue plasminogen activator scores in women with normal pregnancy and patients with non-manifest placental dysfunction $(p>0.05)$. The concentration of plasminogen-1 activator inhibitor in patients with fetal growth retardation exceeded that of women with normal gestation by 1.24 times and reached $76.43 \pm 5.77 \mathrm{ng} / \mathrm{ml}$. Indicators of endotheliotropic marker of vascular thrombogenicity were predominantly predictive, as an additional risk factor for perinatal pathology [1].

During the study, I.B. Borzenko noted activation of the lipid peroxidation system and suppression of the antioxidant protection system in pregnant women with $P D$. A significant $(\mathrm{p}<0.05)$ increase in malondialdehyde level $(7.21 \pm 0.76 \mu \mathrm{mol} / \mathrm{l})$ 
was found in patients with placental dysfunction complicated with fetal growth retardation, compared with malondialdehyde level in pregnant women with physiological gestational course $(3.85 \pm$ $0.51 \mu \mathrm{mol} / \mathrm{l})$. The obtained data on the catalase activity indicated a significant $(\mathrm{p}<0.05)$ decrease in its indicators in patients with PD and FGR to $0.13 \pm 0.03$ c.u. $/ 1$, compared with the same indicators in practically healthy pregnant women $(0.26 \pm 0.0$ c.u./l) [1].

The results of Doppler metamorphosis of the placental fetal blood flow showed that women with placental dysfunction secondary to gestational endotheliopathy had violation of hemodynamic support of the fetus. On week 28 of pregnancy, the patients with FGR had a significant decrease in the pulsation index to $1.62 \pm 0.16 \mathrm{com}$ pared with a similar indicator in pregnant women from the control group $(2.13 \pm 0.14)(\mathrm{p}<0.05)$. These changes in Doppler parameters may be evidence of fetal-placental insufficiency and impaired blood supply to the fetal brain. At the end of the third trimester, a significant decrease $(p<0.05)$ of the pulsation index (to $1.35 \pm 0.17$ ) was found in Doppler ultrasound examination of blood flow of pregnant patients with fetal growth retardation compared with women who had a physiological course of the gestational process $(1.82 \pm 0.11)$. A statistically significant result between women with manifested PD and pregnant women from the control group was also found in terms of GVA $(2.14 \pm 0.5$ and $3.32 \pm 0.31$, respectively).

The labor in pregnant women with PD was associated with an increase in the number of premature births $(\mathrm{p}=0.05)$, cesarean sections $(\mathrm{p}=0.02)$, cases of acute fetal distress $(p=0.01)$, and development of birth abnormalities $(p=0,04)$. The clinically manifested form of PD was the reason for bearing newborns with significantly smaller weight $(\mathrm{p}<0.05)$, lower $(\mathrm{p}<0.05)$ Apgar score at the 1 st and 5th minutes, and an increase in the incidence of adverse perinatal outcomes $(p=0.05)$ compared with women who had physiological pregnancies and deliveries. The infants whose mothers were diagnosed with FGR secondary to endothelial dysfunction also prevailed in frequency.

The evaluation of the morphological features of the placenta in pregnant women with PD of the endothelium showed a decrease in the height of endothelial cells by $4.81 \pm 0.04 \times 10^{-6} \mathrm{~mm}$, a decrease in cell diameter by $3.21 \pm 0.04 \times 10^{-6} \mathrm{~mm}$, an increase in the relative volume of damaged endothelial cells from $6.41 \pm 0.24 \%$ to $48.02 \pm$ $3.63 \%$. The development of FGR in the presence of PD developed secondary to increased damage to the endothelium of the spiral arteries, characterized by the height of endothelial cells $4.04 \pm$ $0.08 \times 10^{-6} \mathrm{~m}$, their diameters $2,92 \pm 0,04 \times 10^{-6} \mathrm{~m}$, the predominance of damaged endothelial cells by $80.57 \pm 0,23 \%$ followed by the development of ischemic lesions and sclerotic changes. Placental vessels in the course of early placental dysfunction were characterized by a $55 \%$ decrease in the area of the lumen accompanied by a decrease in the perimeter and area of these vessels. The endothelial proliferation, hypertrophy of the muscle layer, pronounced growth of perivascular connective tissue with the formation of the socalled "fibrous cuffs" in the myometrial segments of the spiral arteries were also evident. These changes resulted in an additional narrowing of the transparent vessels [1].

In the literature review, Salavati N. et al. [14] intended to give an overview on the clinical relevance of placenta morphometry in the detection of FGR. Future research can focus on the relationship between placental morphometry, FGR and its complications, to improve screening for FGR, and to determine the biological pathways that can be linked to placental dysfunction, in a group of optimally phenotyped cases of FGR.

In the study by Labarrere C.A. et al. [13] analyzing the results of a cross-sectional study of 123 placentas (19-42 weeks' gestation) obtained from normal pregnancies, preterm prelabor rupture of membranes, preterm labor, preeclampsia, intrauterine fetal death, and small for gestational age were noted. They found that $87 \%$ (94/108) of placentas having spiral arteries with failure of physiologic transformation in the basal plate, and $0 \%(0 / 15)$ of placentas having only spiral arteries with complete physiologic transformation, had arterial endothelial and/or interstitial extravillous trophoblasts reactive with the intercellular adhesion molecule- 1 activation marker $(\mathrm{P}<.001)$. A significant correlation $(\mathrm{R} 2=0.84)$ was found between expression of spiral artery endothelial and interstitial extravillous trophoblast intercellular adhesion molecule-1 $(\mathrm{P}<.001)$ in activated placentas.

Korzeniewski SJ. et al. [12] examined of 4006 women with singleton gestations. Maternal plasma angiogenic index-1 ratios were determined using enzyme-linked immunosorbent assays. Maternal plasma angiogenic index-1 (PlGF/sVEGFR-1) is the first biomarker for the burden of placental lesions consistent with maternal vascular underperfusion. They propose that an accumulation of these lesions in placentas 
delivered before 34 weeks is a histologic counterpart of an antiangiogenic profile.

Thus, our clinical prospective study based on a comprehensive assessment of the endothelial component in the development of early PD has shown that gestational endotheliopathy is an independent prognostic factor associated with adverse pregnancy outcomes.

The obtained clinical-morphological and endotheliotropic criteria allowed us to develop and implement a personalized clinical algorithm for managing pregnant women with placental dysfunction. Having analyzed the results of pregnancy in the prospective clinical study, we proved that thanks to the proposed algorithm for personification of the risk of perinatal pathology, it was possible not only to avoid cases of antenatal mortality, but also to prevent intranatal and early neonatal losses in patients with placental dysfunction and fetal growth retardation.

Significant disorders of vasoregulatory, athrombogenic functions, intercellular integration of changes in parameters of uteroplacental-fetal hemodynamics, histomorphometric disorders were identified in pregnant women with PD, which allows better understanding of the pathogenesis of various forms of PD, identifying the ways to predict complications in the early stages of this group of patients. Adequate perinatal support and timely delivery have significantly improved pregnancy outcomes, significantly reduces the number of cases of obstetric impairments.

\section{Declarations}

\section{Statement of Ethics}

The authors have no ethical conflicts to disclosure.

\section{Consent for publication}

All authors give their consent to publication.

Disclosure Statement

Authors have nothing to disclosure.

The manuscript is a part of the thesis of Borzenko I.B.

\section{Funding Sources}

There are no external sources of funding

\section{Data Transparency}

The data can be requested from the authors.

\section{ALGORITHM FOR CLINICAL MANAGEMENT OF PREGNANT WOMEN WITH} EARLY PLACENTAL DYSFUNCTION

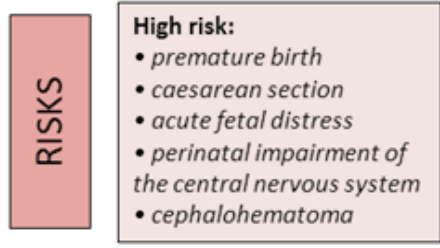

Somatic risk:

- cardiovascular disorders

- varicose veins

- infectious diseases

- dyskinesia of the biliary

tract

\section{Traditional survey}

- conducting biometric tests every 2 weeks

- Doppler examination in the umbilical artery every 2

weeks (from 26-28 weeks)

- Doppler in MCA every 2 weeks (from 32 weeks)

- CTG weekly

-BPP weekly

\section{- determination of the optimal time and method for delivery at}

34-35 weeks

- the optimal delivery time is 36-38 weeks

- FGR is not an indication for caesarean section

- continuous fetal monitoring from the beginning of childbirth

Notes

Notes

MCA - middle cerebral artery; CTG - cardiotocography; BPP - biophysical profile; FGR - fetal growth retardation; MAU - microalbuminuria;

EDVD- endothelium-dependent vasodilation; PI - pulsation index; UA - umbilical arteries; MDA-malonic dialdehyde; DC - diene conjugates;

SOD - superoxide dismutase; TM - thrombomodulin; TPA - tissue plasminogen activator; SVCAM-1 - soluble vascular cell adhesion molecule-1

TS - thrombospondin; SPECAM-1 - soluble platelet-endothelial cell adhesion molecule-1; VEGF - vascular-endothelial growth factor. 


\section{References}

1. Borzenko I. B. Prediction and early diagnosis of fetal growth retardation in pregnant women with placental dysfunction. - Qualifying scientific paper, manuscript. - Kharkiv National Medical University. Kharkiv, $2020-208$ p.

2. Costa M.A. The endocrine function of human placenta: an overview. 2016. Reproductive BioMedicine Online 32 14-43.

3. Figueras F, Gratacos E. An integrated approach to fetal growth restriction. Best Pract Res Clin Obstet Gynaecol 2017;38:48e58.

4. Melnik J.M., Shlyahtina A.A. Early predictors of placental dysfunction. Health of women 2016, 8,25-28.

5. Tomimatsu T., Mimura K., Endo M., Kumasawa K., Kimura T. Pathophysiology of preeclampsia: an angiogenic imbalance and long-lasting systemic vascular dysfunction. Hypertension Research. 2017;40(4):305-310.

6. Ali, S. M., \& Khalil, R. A. Genetic, immune and vasoactive factors in the vascular dysfunction associated with hypertension in pregnancy. 2015. Expert Opinion on Therapeutic Targets, 19, 1495-1515.

7. Anderson NH, Sadler LC, McKinlay CJD, McCowan LME. INTERGROWTH-21st vs customized birthweight standards for identification of perinatal mortality and morbidity. Am J Obstet Gynecol 2016;214. 509.e1e7.

8. Burton GJ, Jauniaux E. Pathophysiology of placental-derived fetal growth restriction. Am J Obstetr Gynecol. 2018;218:S745-61.

9. Flenady V, Wojcieszek AM, Middleton P, et al. Stillbirths: recall to action in high-income countries. Lancet 2016;387:691e702.

10. Lewis AJ, Austin E, Galbally M. Prenatal maternal mental health and fetal growth restriction: a systematic review. J Dev Origins Health Dis. 2016;17:1-13.

11. Menendez-Castro C, Rascher W, Hartner A. Intrauterine growth restriction - impact on cardiovascular diseases later in life. Mol Cell Pediatr. 2018;5:4.

12. Korzeniewski SJ, Romero R, Chaiworapongsa T, et al. Maternal plasma angiogenic index-1 (placental growth factor/soluble vascular endothelial growth factor receptor-1) is a biomarker for the burden of placental lesions consistent with uteroplacental underperfusion: a longitudinal caseecohort study. Am J Obstet Gynecol 2016;214. 629.e1e629.e17.

13. Labarrere, C.A., Dicarlo, H.L., Bammerlin, E. et al, Failure of physiologic transformation of spiral arteries, endothelial and trophoblast cell activation, and acute atherosis in the basal plate of the placenta. Am J Obstet Gynecol. 2017;216:287.e1-287.e16.

14. Salavati N, Smies M, Ganzevoort W, Charles AK, Erwich JJ, Pl?sch T and Gordijn SJ. The Possible Role of Placental Morphometry in the Detection of Fetal Growth Restriction. Front. Physiol. 2019. 9:1884.

15. Rabinovich A, Tsemach T, Novack L, et al. Late preterm and early term: when to induce a growth restricted fetus? A population-based study. J Matern Fetal Neonatal Med 2017 Mar 22:1e7.

16. Gaccioli, F., Lager, S. Placental nutrient transport and intrauterine growth restriction. Front Physiol. 2016;7:40.

17. Sharma et al. Intrauterine growth restriction: antenatal and postnatal aspects. Clinical Medicine Insights: Pediatrics 2016:10 67-83.

18. Baschat AA. Planning management and delivery of the growth-restricted fetus. Best Pract Res Clin Obstetr Gynaecol. 2018;49:53-65.

19. Cuckle H, Maymon R. Development of prenatal screening e a historical overview. Semin Perinatol 2016;40:12e22.

20. Eloundou SN, Lee J, Wu D, Lei J, Feller MC, Ozen M, et al. Placental malperfusion in response to intrauterine inflammation and its connection to fetal sequelae. PLoS ONE 2019;14(4): e0214951

21. Ernst SA, Brand T, Reeske A, Spallek J, Petersen K, Zeeb H. Care-related and maternal risk factors associated with the antenatal nondetection of intrauterine growth restriction: a case-control study from Bremen, Germany. BioMed Res Int. 2017;2017:1746146. 